\title{
A procedure to obtain the parameters of Curie temperature distribution from thermomagnetic and magnetocaloric data
}

A.F. Manchón-Gordón, L.M. Moreno-Ramírez, J.J. Ipus, J.S. Blázquez*, C.F. Conde, V. Franco, A. Conde

Dpto. Física de la Materia Condensada, ICMSE-CSIC, Universidad de Sevilla, P.O. Box 1065, 41080 Sevilla, Spain

"The corresponding author e-mail: jsebas@us.es

ABSTRACT: We propose a procedure for the determination of the parameters of the Curie temperature distribution (TCD) in a compositionally inhomogeneous ferromagnetic material. Assuming a Gaussian TCD and using a mean field approach based on the Brillouin function, we report that with respect to the average value of the distribution: a) both inflection point of magnetization, $T_{\text {inf }}$, and temperature at maximum magnetic entropy change curves, $T_{p k}^{M C E}$, shift to lower temperatures and $\mathrm{b}$ ) temperature at maximum paramagnetic susceptibility, $T_{p k}^{\chi}$, shifts to higher temperatures. Using these evolutions as a function of the TCD broadening and fitting them to a second order polynomial function, a self-consistent procedure to determine the parameters of the distribution is supplied. These predictions have been experimentally tested for a ball milled $\mathrm{Fe}_{70} \mathrm{Zr}_{30}$ amorphous alloy using thermomagnetic and magnetocaloric measurements. The obtained parameters using the proposed procedure agree with those directly measured using Mössbauer spectrometry.

KEYWORDS: Curie temperature distribution, inhomogeneous materials, magnetocaloric effect, law of approach to saturation

\section{INTRODUCTION}


The determination of the Curie temperature, $T_{C}$, defined as the temperature at which a ferromagnetic material loses its spontaneous magnetization, is not trivial. It is usually approximated to the inflection point, $T_{\text {inf }}$, at low fields, of the magnetization curves as a function of temperature and magnetic field $M(T, H)$. However, the obtained value of $T_{\text {inf }}$ is field dependent.

On the other hand, the knowledge of the physics underlying magnetocaloric effect (MCE) [1-5], defined as the adiabatic/isothermal change of the temperature/magnetic entropy of a system due to a change in the applied magnetic field $\left(\Delta T_{a d}\right.$ and $\Delta S_{M}$, respectively), has allowed the study of several fundamental aspects of the magnetic transitions such as critical exponents [6,7], nature of the transition [8] or accurate determination of Curie temperature [9]. In this sense, $T_{C}$ can be approximated to the temperature at which magnetic entropy change has its peak value, $T_{p k}^{M C E}$. However, it has been shown that both $T_{C}$ and $T_{p k}^{M C E}$ are not necessarily coincident and the deviation depends on the field [10].

Therefore, although except in the case of mean field theories $[9,10]$ both temperatures, $T_{\text {inf }}$ and $T_{p k}^{M C E}$, are not strictly equal to the actual $T_{C}$, they have been used to determine it as the differences can be below the experimental error under conventional measuring conditions. However, experimental differences between $T_{i n f}$ and $T_{p k}^{M C E}$ have been observed not only for non-mean field systems, but even in systems with compositional inhomogeneities and/or structural defects $[11,12]$ that lead to a broad Curie temperature distribution [13]. Moreover it has been shown, by numerical modeling, how a normal distribution of Curie temperatures can shift the peaks of specific heat capacity and the adiabatic temperature [14] and the magnetic entropy change [15] to lower temperatures with respect to the average value of the distribution. 
In fact, many production techniques lead to the formation of a non-perfectly homogeneous system. Indeed, certain compositional heterogeneity can be expected leading to a distribution of the physical properties rather than a sharp behavior characteristic of the theoretically pure system. This particularly affects the interpretation of amorphous samples or crystalline phases with broad compositional ranges. The knowledge of the parameters characterizing such distributions can help to reproduce a more realistic description of the samples' behavior.

Previous works using a distribution of Curie temperatures obtain the corresponding parameters (average value of the Curie temperature, $\overline{T_{C}}$, and its standard deviation, $\Delta T_{C}$ ) from a direct fitting of the temperature variation of the magnetization [16]. In this work, we show that even in the case of mean field approach, when a distribution of Curie temperatures is considered, not only $T_{p k}^{M C E}$ but also $T_{\text {inf }}$ and the temperature at which paramagnetic susceptibility has its peak value, $T_{p k}^{\chi}$, shift with respect to the average value of the Curie temperature distribution. This allows us to propose a quantitative procedure to obtain the parameters of the distribution using thermomagnetic and magnetocaloric measurements of the sample under study. The procedure is developed under the assumptions of the Brillouin equation of state and a Gaussian distribution of Curie temperatures. The applicability of the procedure is tested after a comparative study with the experimental results obtained for an amorphous $\mathrm{Fe}_{70} \mathrm{Zr}_{30}$ alloy prepared by mechanical alloying.

\section{Model based on a Gaussian distribution of Curie temperatures}

The proposed model should describe the behavior of the magnetization, magnetic susceptibility and MCE response in a compositionally inhomogeneous ferromagnetic 
material. Assuming the existence of a non-unique Curie temperature, the Curie distribution has been described using a Gaussian distribution as:

$$
\rho\left(T_{C}, \overline{T_{C}}, \Delta T_{C}\right)=\frac{1}{\sqrt{2 \pi} \Delta T_{C}} \exp \left(-\frac{1}{2} \frac{\left(T_{C}-\bar{T}_{C}\right)^{2}}{\Delta T_{C}^{2}}\right)
$$

where $\overline{T_{C}}$ is the average value of the Curie temperature and $\Delta T_{C}$ is the width of the distribution (standard deviation), respectively.

Using this distribution of Curie temperatures, magnetization can be obtained by a superposition of individual Brillouin functions, $M_{S}^{\text {ideal }}$ :

$$
M\left(H, T, \overline{T_{C}}, \Delta T_{C}\right)=\sum_{T_{C}=1 \mathrm{~K}}^{\overline{T_{C}}+\epsilon} M_{S}^{\text {ideal }}\left(H, T, T_{C}\right) \rho\left(T_{C}, \overline{T_{C}}, \Delta T_{C}\right)
$$

where $\epsilon$ is the range of the distribution used in the calculation; beyond $\overline{T_{C}}+\epsilon$ those contributions to the total magnetization are neglected.

As temperature increases, some contributions become paramagnetic. The paramagnetic susceptibility $\chi$ for a single ideal contribution is zero for $T<T_{C}$ and follows the CurieWeiss law for $T>T_{C}$. Thus the general behavior of paramagnetic susceptibility has been built using a superposition of these contributions:

$$
\chi\left(H, T, \overline{T_{C}}, \Delta T_{C}\right)=\sum_{T_{C}=1 \mathrm{~K}}^{\overline{T_{C}}+\epsilon} \chi_{\text {ideal }}\left(H, T, T_{C}\right) \rho\left(T_{C}, \overline{T_{C}}, \Delta T_{C}\right)
$$

On the other hand, using the same idea, the magnetic entropy change can be expressed as:

$$
\Delta S_{M}\left(H, T, \overline{T_{C}}, \Delta T_{C}\right)=\sum_{T_{C}=1 \mathrm{~K}}^{\overline{T_{C}}+\epsilon} \Delta S_{M}^{i d e a l}\left(H, T, T_{C}\right) \rho\left(T_{C}, \overline{T_{C}}, \Delta T_{C}\right)
$$

The expressions described above were computed using Matlab for different values of $\Delta T_{C}$ and for $\overline{T_{C}}=300 \mathrm{~K}$. In order to simplify the simulations, the same value of magnetic moment has been considered for each contribution. It has to be noticed that room 
temperature is assumed in a first instance, $\epsilon=200 \mathrm{~K}$ has been selected and the $\Delta T_{C}$ values explored here are well below this value.

Figure 1 shows a) simulated $\mathrm{d} M(T) / \mathrm{d} T$ curves at $\mu_{0} H=0 \mathrm{~T}$ using the derivative of Eq. 3, b) $\chi(T)$ curves obtained from Eq. 4 and c) the simulated $\Delta S_{M}(T)$ curves at $\mu_{0} \Delta H=1 \mathrm{~T}$ obtained from Eq. 5, as a function of the temperature for different values of $\Delta T_{C}$. With the increasing of $\Delta T_{C}$ the curves become broader and a shift of the peak temperature can be observed. While the inflection point in the temperature dependence of magnetization, $T_{i n f}$, and the temperature corresponding to the maximum magnetocaloric effect, $T_{p k}^{M C E}$, shift to lower temperatures, the temperature of the peak of paramagnetic susceptibility $T_{p k}^{\chi}$ shifts to higher temperatures with respect to $\overline{T_{C}}$.

The evolution of the peak temperatures of these magnitudes can be fitted to a second order polynomial function as a function of the broadening of the Curie temperature distribution as (see insets Fig. 1):

$$
\begin{gathered}
T_{\text {inf }}-\overline{T_{C}}=-1.056(5) \Delta T_{C}+0.0026(3) \Delta T_{C}{ }^{2} \\
T_{p k}^{\chi}-\overline{T_{C}}=0.571(9) \Delta T_{C}+0.0043(2) \Delta T_{C}{ }^{2} \\
T_{p k}^{M C E}-\overline{T_{C}}=-0.709(15) \Delta T_{C}+0.0014(4) \Delta T_{C}{ }^{2}
\end{gathered}
$$

Where the least square regression yields correlation coefficients of $r^{2}=0.9998,0.9999$ and 0.9995 for Eq. 6, 7 and 8, respectively and the errors are taken as the standard deviation. The coefficients in equations above do not depend on $\overline{T_{C}}$ in the explored range $\left(200 \leq \overline{T_{C}} \leq 400 \mathrm{~K}\right)$ and field dependences are avoided in our calculations using a maximum applied field of $\mu_{0} \Delta H=1 \mathrm{~T}$ for the calculation of $\Delta S_{M}(T)$. Eqs. 6, 7 and 8 yield $T_{\text {inf }}<T_{p k}^{M C E}<\overline{T_{C}}<T_{p k}^{\chi}$. These results show that the use of both, the inflection point in the temperature dependence of magnetization and the temperature at which $\left|\Delta S_{M}\right|$ is maximum to determine the Curie temperature leads to an underestimation of 
the average value of the distribution. Our simulations indicate that linear approaches should be good enough for $\Delta T_{C}<40 \mathrm{~K}$ and simplify equations (6) to (8) as:

$$
\begin{aligned}
& T_{\text {inf }}-\overline{T_{C}} \sim-1.017(6) \Delta T_{C}\left(r^{2}=0.9997\right) ; T_{p k}^{\chi}-\overline{T_{C}} \sim 0.455(14) \Delta T_{C}\left(r^{2}=\right. \\
& 0.992) \text { and } T_{p k}^{M C E}-\overline{T_{C}} \sim-0.686(7) \Delta T_{C}\left(r^{2}=0.9995\right) .
\end{aligned}
$$

\section{Comparison to experimental results}

In order to experimentally test the validity of the results derived from the proposed model, an amorphous alloy with $\mathrm{Fe}_{70} \mathrm{Zr}_{30}$ at.\% composition was prepared by ball milling for $50 \mathrm{~h}$ at $350 \mathrm{rpm}$. The alloy phase structure was characterized by X-ray diffraction (Bruker D8 I, Cu-K $\alpha$ radiation) and by ${ }^{57} \mathrm{Fe}$ Mössbauer spectroscopy in transmission geometry using a ${ }^{57} \mathrm{Co}(\mathrm{Rh})$ source. Further milling parameters and a detailed microstructural evolution of the sample can be found elsewhere [17]. Magnetization curves were measured as a function of temperature using a maximum applied field of $\mu_{0} H=1.5 \mathrm{~T}$ in a Lakeshore 7407 vibrating sample magnetometer (resolution $2.5 \mu \mathrm{emu}$ ). A Mettler Toledo XP 26 microbalance (resolution of $0.001 \mathrm{mg}$ ) was used to weight the samples. Saturation magnetization, $M_{S}$, and paramagnetic susceptibility, $\chi$, were obtained by fitting the experimental high-field magnetization curve to the law of approach to saturation [18]:

$$
M=M_{S}\left(1-\frac{a}{H}-\frac{b}{H^{2}}\right)+\chi H
$$

where $H$ is the internal field (demagnetizing field was corrected using a density of 7205 $\mathrm{kg} / \mathrm{m}^{3}$ ). Theoretically in Eq. 9, the value of the parameter $a$ is a measure of the structural inhomogeneities caused by defects within magnetic substances $[19,20]$ and $b$ is related to the effective anisotropy [21]. Least square regression was applied to Eq. 9 
for $M / M_{S}$ values larger than 0.8. Magnetic entropy change has been calculated from isothermal magnetization curves performed with the help of the Magnetocaloric Effect Analysis Program [22]. Details of the correction of the demagnetizing factor and the MCE of the sample can be found elsewhere [23]. Figure 2 shows $\mathrm{d} M_{S} / \mathrm{d} T, \chi$ and $\Delta S_{M}$ (at $\mu_{0} \Delta H=1 \mathrm{~T}$ ) for the studied sample with their corresponding peak temperature values.

Combining Eqs. 6, 7 and 8 and using the experimental values $\left(T_{\text {inf }}=230 \pm 3 \mathrm{~K}\right.$, $T_{p k}^{M C E}=237.5 \pm 2.5 \mathrm{~K}$ and $T_{p k}^{\chi}=265 \pm 3 \mathrm{~K}$ ), the values of $\overline{T_{C}}$ and $\Delta T_{C}$ can be obtained. For example, using Eqs. 6 and 7, $\Delta T_{C}=24 \pm 5 \mathrm{~K}$. The same result is obtained with the other possible combinations of equations. On the other hand, introducing the calculated value of $\Delta T_{C}$ in one of the previous equations, $\overline{T_{C}}=254 \pm 4 \mathrm{~K}$ is obtained.

The distribution of Curie temperatures was experimentally measured from the thermal evolution of the paramagnetic fraction in Mössbauer spectra (Fig. 3). Two contributions were used to fit the experimental spectra: a small ferromagnetic site at $\sim 33 \mathrm{~T}$ and a distribution of hyperfine fields (HFD). The discrete sextet represents the Fe environment in residual $\alpha$-Fe bcc phase ( $\sim 3 \%$ of Fe atoms). In the case of very low temperatures, the HFD is ascribed to Fe atoms in the amorphous phase with a lower Curie temperature than $\alpha-\mathrm{Fe}$ and thus with lower hyperfine field contributions $(\mathrm{HF}<33$ T). As temperature increases, low field contributions collapse to a peak centered at HF 3 T. This peak actually corresponds to paramagnetic contributions. A more realistic model should fit these contributions using a quadrupolar distribution [17]. However, in order to avoid the errors derived from overlapping and taking into account that we are only interested in the fraction area, we have used the area of this low HF peak to account for the paramagnetic fraction of the sample. Moreover, the use of a unique distribution allows us to obtain a continuous description of the evolution of the Mössbauer spectra as a function of temperature. 
The thermal evolution of the hyperfine field distributions $\mathrm{P}\left(\mathrm{B}_{\mathrm{hf}}\right)$ is shown in Fig. 4a. These $\mathrm{P}\left(\mathrm{B}_{\mathrm{hf}}\right)$ were fitted using three Gaussians distributions, one at $\mathrm{HF} \sim 3 \mathrm{~T}$ (attributed to paramagnetic contribution) and two other assigned to truly ferromagnetic sites. The behavior of $\mathrm{P}\left(\mathrm{B}_{\mathrm{hf}}\right)$ (continuous and not abrupt transition above inflection point of the thermomagnetic curves $\sim 230 \mathrm{~K}$ ) has been previously reported in $\mathrm{Fe}-\mathrm{Zr}$ alloys prepared by ball milling [24] and other techniques $[25,26]$. Figure $4 \mathrm{~b}$ shows the variation of the relative area of the paramagnetic component to the total area of $\mathrm{P}\left(\mathrm{B}_{\mathrm{hf}}\right)$ as a function of temperature. This component, $X_{\text {para }}$, has been fitted using the cumulative distribution function:

$$
X_{\text {para }}=\frac{1}{2} \operatorname{erfc}\left(\frac{\overline{T_{C}}-T}{\sqrt{2} \Delta T_{C}}\right)
$$

where erfc is the complementary error function. From the least square fitting to the data of figure $4 \mathrm{~b}$, the width and the average value of the Curie temperature distribution can be estimated as $\Delta T_{C}=31 \pm 9 \mathrm{~K}$ and $\overline{T_{C}}=252 \pm 5 \mathrm{~K}$. These values are in good agreement with the values obtained from the proposed procedure $\left(\Delta T_{C}=24 \pm 5 \mathrm{~K}\right.$ and $\left.\overline{T_{C}}=254 \pm 4 \mathrm{~K}\right)$ and supported it. Deviation found in the width of the distribution could be ascribed to a departure from mean field behavior of the system (both $T_{i n f}$ and $T_{p k}^{M C E}$ should shift to higher values [9]). On the other hand, considering Heisenberg critical exponents for a near neighbors interacting system, Arrot-Noakes equation of state [27], and limiting $\Delta T_{C}$ to below $40 \mathrm{~K}$, equations (6) and (7) for the shift of the peak temperatures are modified to:

$$
\begin{gathered}
\left(T_{\text {inf }}\right)_{H b}-\overline{T_{C}} \sim(-0.743 \pm 0.009) \Delta T_{C} \\
\left(T_{p k}^{\chi}\right)_{H b}-\overline{T_{C}} \sim(0.31 \pm 0.02) \Delta T_{C}-(0.0035 \pm 0.0006) \Delta T_{C}{ }^{2}
\end{gathered}
$$


with $r^{2}=0.999$ and 0.994 , respectively. The subindex $\mathrm{Hb}$ indicates that a Heisenberg system is assumed. It is worth noticing that, in this case, the shift of both $\left(T_{\text {inf }}\right)_{H b}$ and $\left(T_{p k}^{\chi}\right)_{H b}$ is lower with respect $\overline{T_{C}}$. Using Eqs. (11) and (12) the parameters of the distribution can be estimated as $\left(\Delta T_{C}\right)_{H b}=38 \pm 7 \mathrm{~K}$ and $\left(\overline{T_{C}}\right)_{H b}=258 \pm 8 \mathrm{~K}$. This leads to higher values than those derived from the proposed model using Brillouin equation and those derived from Mössbauer spectra are found in between the two models: long range interaction (mean field) and first neighbor interaction (Heisenberg), as expected.

Figure 5 shows the experimental $M_{S}(T)$ data along with the simulated curves generated by Eq. 3 using the values determined from the fitting of $X_{\text {para }}$ (left) and those derived from the proposed procedure (right). The extreme curves defined by the error bars of the parameters of the distribution are also shown. The value of magnetic moment used in the simulations, $\mu_{F e_{70} Z r_{30}}=1.05 \mu_{B}$, is the only free parameter and it is similar but below that found in the literature $\left(1.19 \mu_{B}\right)$ [24]. Moreover, the $\sim 3 \% \mathrm{Fe}$ atoms into the residual $\alpha-\mathrm{Fe}_{95} \mathrm{Zr}_{5}$ phase detected from Mössbauer spectroscopy [17] has been considered in the calculations assuming a magnetic moment of $2.2 \mu_{B}$ per Fe atom. A good agreement between the experimental data and the simulated curves can be observed.

\section{Conclusions}

In this work, the thermomagnetic and magnetocaloric response in a compositionally inhomogeneous ferromagnetic material, characterized by a non-negligible distribution of Curie temperatures, are reproduced assuming a Gaussian distribution of the Curie temperatures and using a mean field approach based on the Brillouin function. We show that both the inflection point in the temperature dependence of magnetization and the temperature corresponding to the maximum magnetocaloric effect shift to lower 
temperatures, while the temperature of the peak of paramagnetic susceptibility shifts to higher temperatures in such a case. These shifts can be fitted to a second order polynomial which supplies a quantitative procedure to identify $\overline{T_{C}}$ and the width of the distribution in an compositional inhomogeneous sample. The results have been tested by comparing with the experimental results of Mössbauer spectrometry as a function of temperature for an amorphous $\mathrm{Fe}_{70} \mathrm{Zr}_{30}$ alloy prepared by ball milling. Using this simple model, we succeed reproducing the results obtained from experiments.

\section{Acknowledgements}

This work was supported by AEI/FEDER-UE (Project MAT 2016-77265-R) and the PAI of the Regional Government of Andalucía. A.F. Manchón-Gordón acknowledges a VPPI-US fellowship. L.M Moreno-Ramírez acknowledges a FPU fellowship from the Spanish MECD. 


\section{References}

[1] Gschneidner KA, Pecharsky VK. Magnetocaloric materials. Annual Review of Materials Science 2000;30:387-429. https://doi.org/10.1146/annurev.matsci.30.1.387

[2] Bruck E, Tegus O, Thanh DTC, Buschow KHJ. Magnetocaloric refrigeration near room temperature. Journal of Magnetism and Magnetic Materials 2007;310:2793-9. https://doi.org/10.1016/i.jmmm.2006.10.1146

[3] Gutfleisch O, Willard MA, Bruck E, Chen CH, Sankar SG, Liu JP. Magnetic Materials and Devices for the 21st Century: Stronger, Lighter, and More Energy Efficient. Advanced Materials 2011;23:821-42. https://doi.org/10.1002/adma.201002180

[4] Franco V, Blazquez JS, Ingale B, Conde A. The Magnetocaloric Effect and Magnetic Refrigeration Near Room Temperature: Materials and Models. Annual Review of Materials Research2012. p. 305-42. https://doi.org/10.1146/annurev-matsci-062910-100356

[5] Franco V, Blazquez JS, Ipus JJ, Law JY, Moreno-Ramírez LM, Conde A. Magnetocaloric effect: from materials research to refrigeration devices. Progress in Materials Science 2018;93:112232. https://doi.org/10.1016/i.pmatsci.2017.10.005

[6] Franco V, Blazquez JS, Conde A. Field dependence of the magnetocaloric effect in materials with a second order phase transition: A master curve for the magnetic entropy change. Applied Physics Letters 2006;89:222512. https://doi.org/10.1063/1.2399361

[7] Franco V, Conde A. Scaling laws for the magnetocaloric effect in second order phase transitions: From physics to applications for the characterization of materials. International Journal of Refrigeration-Revue Internationale Du Froid 2010;33:465-73. https://doi.org/10.1016/j.ijrefrig.2009.12.019

[8] Law JY, Franco V, Moreno-Ramírez LM, Conde A, Karpenkov DY, Radulov I, et al. A quantitative criterion for determining the order of magnetic phase transitions using the magnetocaloric effect. Nature communications 2018;9:2680. https://doi.org/10.1038/s41467018-05111-w

[9] Moreno-Ramirez LM, Blazquez JS, Franco V, Conde A, Marsilius M, Budinsky V, et al. A New Method for Determining the Curie Temperature From Magnetocaloric Measurements. leee Magnetics Letters 2016;7:1-4. https://doi.org/10.1109/LMAG.2016.2533481

[10] Franco V, Conde A, Kuz'min MD, Romero-Enrique JM. The magnetocaloric effect in materials with a second order phase transition: Are T-C and T-peak necessarily coincident? Journal of Applied Physics 2009;105:07A917 https://doi.org/10.1063/1.3063666

[11] Kuz'min MD, Richter $M$, Tishin AM. Field dependence of magnetic entropy change: Whence comes an intercept? Journal of Magnetism and Magnetic Materials 2009;321:L1-L3. https://doi.org/10.1016/j.jmmm.2008.07.004

[12] Moreno-Ramirez LM, Ipus JJ, Franco V, Blazquez JS, Conde A. Analysis of magnetocaloric effect of ball milled amorphous alloys: Demagnetizing factor and Curie temperature distribution. Journal of Alloys and Compounds 2015;622:606-9. https://doi.org/10.1016/j.jallcom.2014.10.134

[13] Alvarez-Alonso P, Llamazares JLS, Sanchez-Valdes CF, Cuello GJ, Franco V, Gorria P, et al. On the broadening of the magnetic entropy change due to Curie temperature distribution. Journal of Applied Physics 2014;115:17A929. https://doi.org/10.1063/1.4867346

[14] Bahl CRH, Bjork R, Smith A, Nielsen KK. Properties of magnetocaloric materials with a distribution of Curie temperatures. Journal of Magnetism and Magnetic Materials 2012;324:564-8. https://doi.org/10.1016/i.jmmm.2011.08.044

[15] Amaral JS, Tavares PB, Reis MS, Araujo JP, Mendonca TM, Amaral VS, et al. The effect of chemical distribution on the magnetocaloric effect: A case study in second-order phase transition manganites. Journal of Non-Crystalline Solids 2008;354:5301-3. https://doi.org/10.1016/j.jnoncrysol.2008.05.078 
[16] Campillo G, Berger A, Osorio J, Pearson JE, Bader SD, Baca E, et al. Substrate dependence of magnetic properties of $\mathrm{La} 0.67 \mathrm{Ca} 0.33 \mathrm{MnO} 3$ films. Journal of Magnetism and Magnetic Materials 2001;237:61-8. https://doi.org/10.1016/S0304-8853(01)00482-6

[17] Manchon-Gordon AF, Ipus JJ, Blazquez JS, Conde CF, Conde A. Evolution of Fe environments and phase composition during mechanical amorphization of Fe70Zr30 and Fe70Nb30 alloys. Journal of Non-Crystalline Solids 2018;494:78-85. https://doi.org/10.1016/j.jnoncrysol.2018.04.061

[18] Coey JMD. Manetism and magnetic material. New York: Cambridge University Press; 2010. [19] Brown WF. Theory of the approach to magnetic saturation. Physical Review 1940;58:73643. https://doi.org/10.1103/PhysRev.58.736

[20] Brown WF. The effect of dislocations on magnetization near saturation. Physical Review 1941;60:139-47. https://doi.org/10.1103/PhysRev.60.139

[21] Xiong $X Y$, Finlayson TR, Muddle $B C$. The approach to saturation magnetization of nanocrystalline Fe90Zr7B3 alloy. Journal of Physics D-Applied Physics 2001;34:2845-53. https://doi.org/10.1088/0022-3727/34/18/319

[22] http://www.lakeshore.com/products/Vibrating-Sample-Magnetometer/Pages/MCE.aspx [23] Manchón-Gordón AF, Ipus JJ, Moreno-Ramírez LM, Blázquez JS, Conde CF, Franco V, et al. Correction of the shape effect on magnetic entropy change in ball milled Fe70Zr30 alloys. Journal of Alloys and Compounds 2018;765:437-43. https://doi.org/10.1016/j.jallcom.2018.06.176

[24] Pizarro R, Garitaonandia JS, Plazaola F, Barandiaran JM, Greneche JM. Magnetic and Mossbauer study of multiphase Fe-Zr amorphous powders obtained by high energy ball milling. Journal of Physics-Condensed Matter 2000;12:3101-12. https://doi.org/10.1088/0953$8984 / 12 / 13 / 318$

[25] Unruh KM, Chien CL. MAGNETIC-PROPERTIES AND HYPERFINE INTERACTIONS IN AMORPHOUS FE-ZR ALLOYS. Physical Review B 1984;30:4968-74. https://doi.org/10.1103/PhysRevB.30.4968

[26] Kaul S, Siruguri V, Chandra G. Magnetization and Mossbauer study of the reentrant amorphous Fe90Zr10 alloy. Physical Review B 1992;45:12343-56. https://doi.org/10.1103/PhysRevB.45.12343

[27] Arrott A, Noakes JE. APPROXIMATE EQUATION OF STATE FOR NICKEL NEAR ITS CRITICAL TEMPERATURE. Physical Review Letters 1967;19:786-9.

https://doi.org/10.1103/PhysRevLett.19.786 


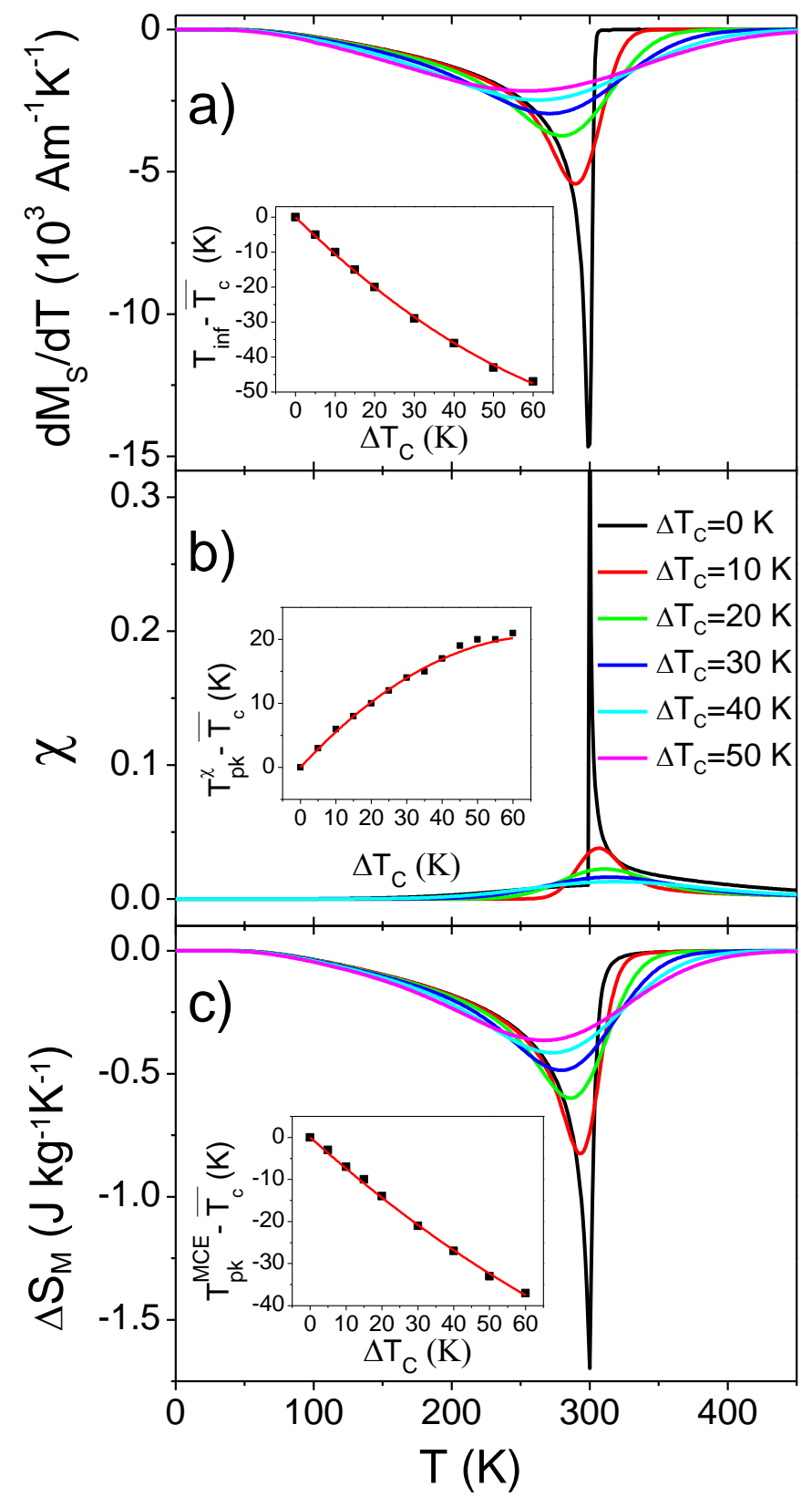

Figure 1. a) Simulated $\mathrm{d} M / \mathrm{d} T$ curves obtained from derivative of Eq. 3 for different values of $\Delta T_{C}$. Inset: dependence of $T_{\text {inf }}-\overline{T_{C}}$ with $\Delta T_{C}$. b) Simulated paramagnetic susceptibility obtained from Eq. 4. Inset: dependence of $T_{p k}^{\chi}-\overline{T_{C}}$ with $\Delta T_{C}$. c) Simulated magnetic entropy change curves at $\mu_{0} \Delta H=1 \mathrm{~T}$ obtained from Eq. 5. Inset: dependence of $T_{p k}^{M C E}-\overline{T_{C}}$ with $\Delta T_{C}$. All the curves have been obtained assuming that $\overline{T_{C}}=300 \mathrm{~K}$. 


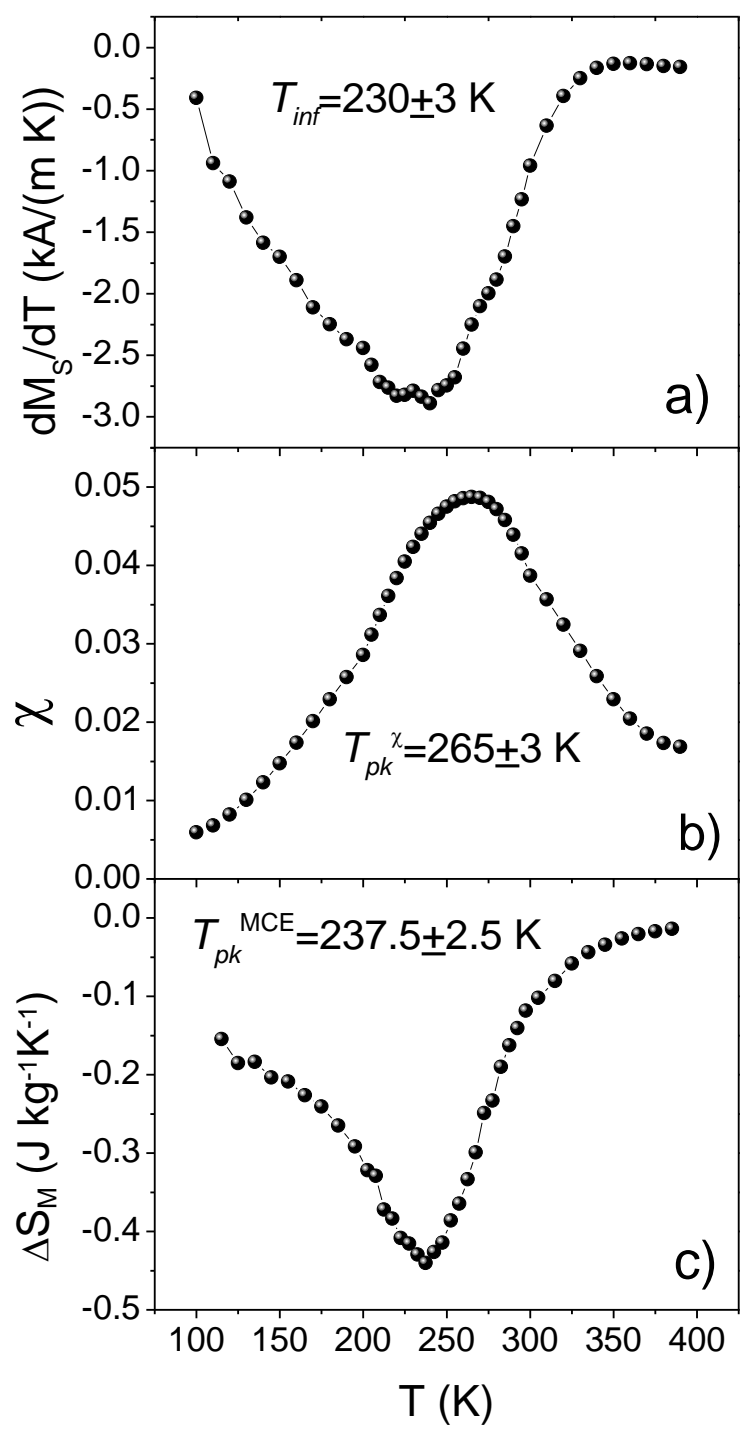

Figure 2. Experimental results for mechanically alloyed $\mathrm{Fe}_{70} \mathrm{Zr}_{30}$ amorphous alloy. a) $\mathrm{d} M / \mathrm{d} T$ and b) paramagnetic susceptibility curves from the law of approach to saturation. c) Magnetic entropy change curve at $\mu_{0} \Delta H=1 \mathrm{~T}$. 


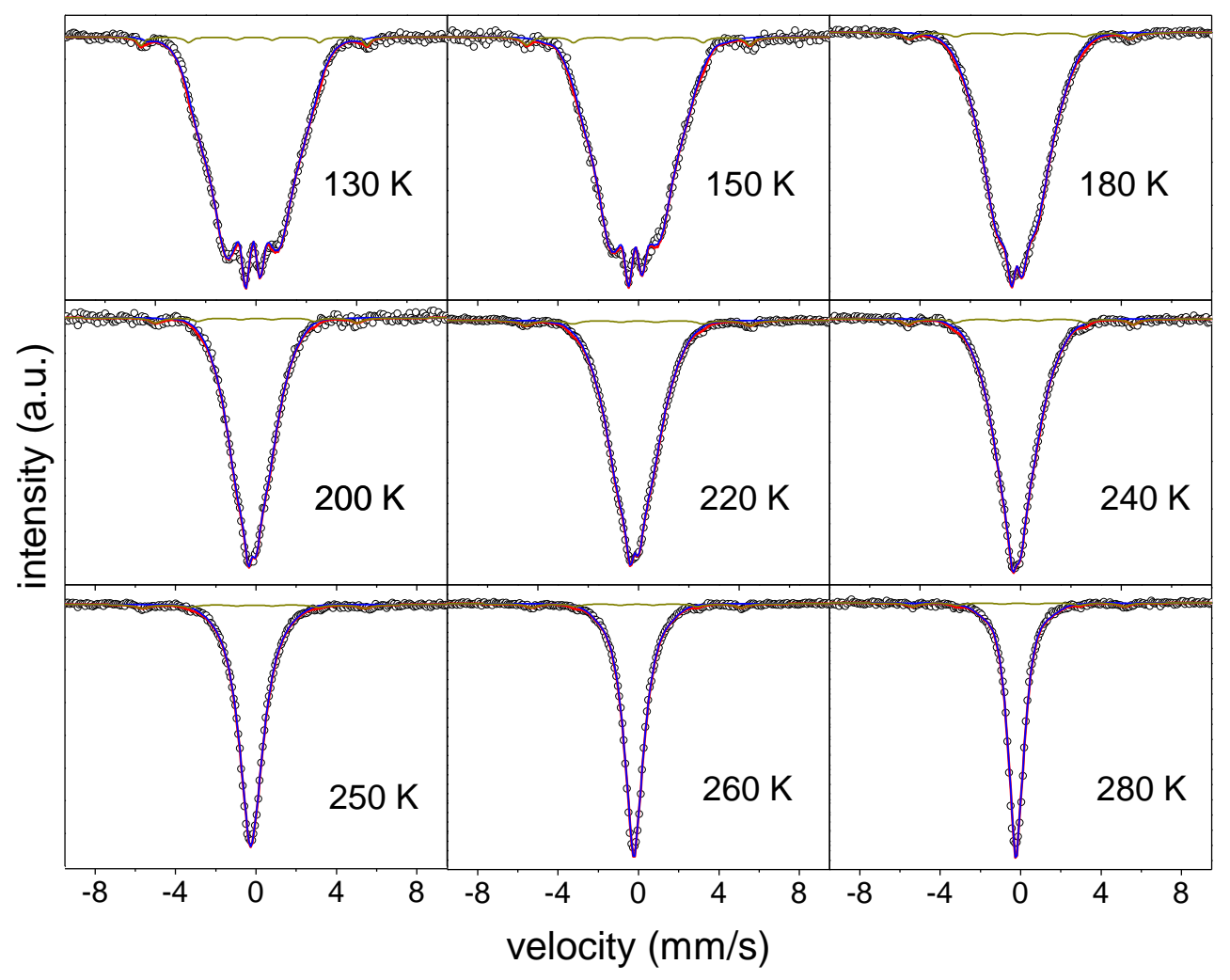

Figure 3. Mössbauer spectra of $\mathrm{Fe}_{70} \mathrm{Zr}_{30}$ mechanically alloyed samples at various temperatures. The symbols correspond to the experimental data. Magenta line corresponds to the total fitting and the small green contribution to the remnant $\alpha$-Fe crystallites. 

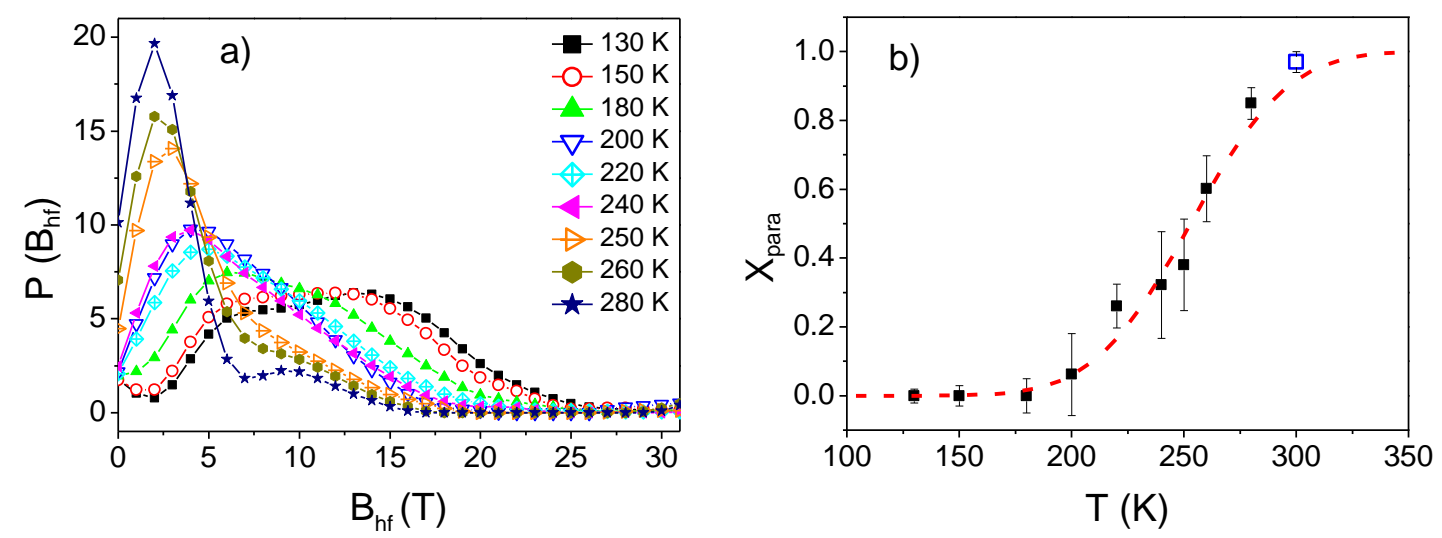

Figure 4. a) Probability distribution of hyperfine field distribution for the studied sample as a function of temperature. b) Evolution with temperature of the paramagnetic contribution. The hollow symbol corresponds to room temperature measurement from [17]. The discontinuous line corresponds to the fit of experimental data using the cumulative distribution function. 


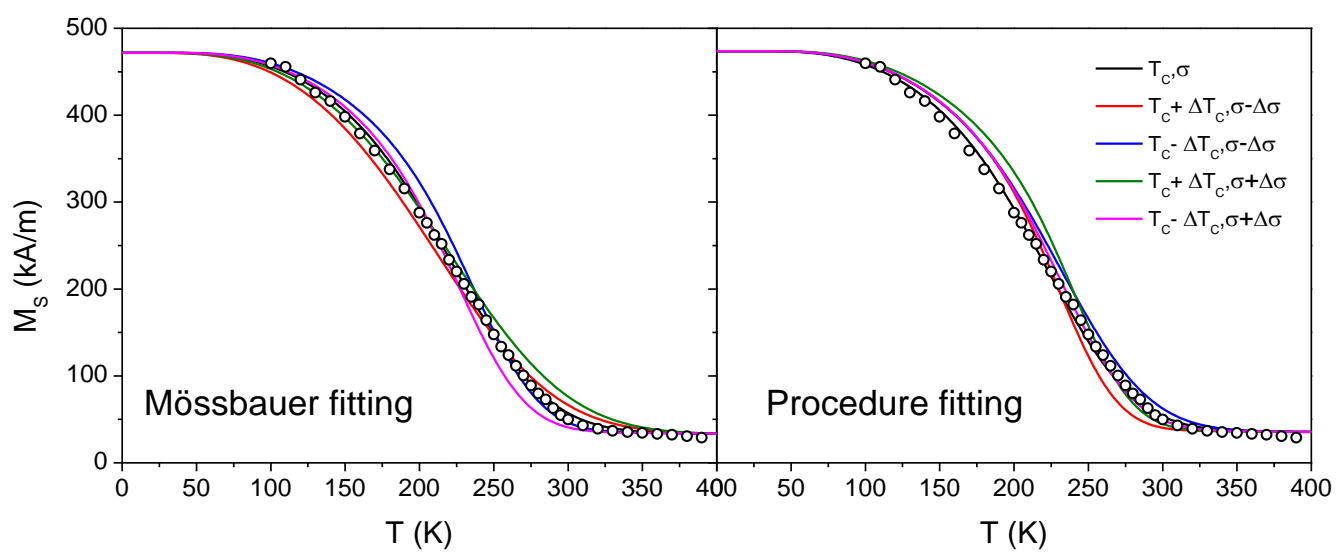

Figure 5. Experimental data (symbols) and simulated curves obtained from Mössbauer spectra (left) and from the procedure proposed here (right). The extreme curves defined by the error bars of the corresponding values of mean Curie temperature $\left(\overline{T_{C}}=252 \pm 5 \mathrm{~K}\right.$, left, $254 \pm 4 \mathrm{~K}$, right) and width of the distribution are also shown. $\left(\Delta T_{C}=31 \pm 9 \mathrm{~K}\right.$, left, and $24 \pm 5 \mathrm{~K}$, right) 
Journal of Non-Crystalline Solids 520 (2019) 119460 Correction. Journal of Non-

Crystalline Solids 538 (2020) 120047

Correction to "A procedure to obtain the parameters of Curie temperature distribution

from thermomagnetic and magnetocaloric data" orginally published as J. Non-Cryst.

Solids 520, 119460 (2019)

A.F. Manchón-Gordón, L.M. Moreno-Ramírez, J.J. Ipus, J.S. Blázquez*, C.F. Conde, V. Franco,

\section{A. Conde}

*Corresponding author e-mail: jsebas@us.es

We have detected a mistake in the published paper [1]. A factor of $\sqrt{2}$ was omitted in the Gaussian expansion used for the simulations. This implies that $\Delta T_{C}$ in equations (5), (6), (13) and (14) must be substituted by $\sqrt{2} \Delta T_{C}$. As a consequence, the $\mathrm{X}$-axis of the insets of Figure 1 (a) and (b) in [1] correspond to $\Delta T_{C} / \sqrt{2}$. The new values derived are $\Delta T_{C}=32 \pm 4 \mathrm{~K}$ and $\overline{T_{C}}=253 \pm 3 \mathrm{~K}$. It is worth mentioning that the mistake found does not affect the conclusions presented in our previous work but the agreement between the experimental data and the fitted curve is improved. Figure 1 is the corrected version of figure 5 (right) in [1]. As observed, the fitting is improved.

\section{References}

[1] A.F. Manchón-Gordón, L.M. Moreno-Ramírez, J.J. Ipus, J.S. Blázquez, C.F. Conde, V. Franco, A. Conde "A procedure to obtain the parameters of Curie temperature distribution from thermomagnetic and magnetocaloric data” J. Non-Cryst. Solids, 520 (2019) 119460 